\title{
ОПРЕДЕЛЕНИЕ ТОЛЩИНЫ ПЛЕНОК МЕТОДОМ СПЕКТРОСКОПИЧЕСКОЙ РЕФЛЕКТОМЕТРИИ С ИСПОЛЬЗОВАНИЕМ АЛГОРИТМОВ БЫСТРОГО ПРЕОБРАЗОВАНИЯ ФУРЬЕ
}

\section{FILM THICKNESS DETERMINATION BY SPECTROSCOPIC REFLECTOMETRY METHOD USING FOURIER FAST TRANSFORM ALGORITHMS}

\begin{abstract}
Л.Асиновский', Ph.D, Д.Никулин², инженер по наладке и испытаниям, (ORCID: 0000-0003-0265-561X), B.Васильев², к.т.н., технический директор компании ЗЭНКО ПЛАЗМА, (ORCID: 0000-0002-0976-7852) / vladimir. vasilyev@zencoplasma.ru L.Asinovski', Ph.D, D.Nikulin², Bachelor of Engineering and Technology, Commissioning and Testing Engineer, V.Vasiljev², Cand. of Sc. (Engineering), Chief Technical Officer
\end{abstract}

DOI: 10.22184/1993-8578.2019.12.5.260.267

Получено: 03.04.2019 г.

В работе приведены примеры применения программного пакета обработки отраженных спектров для определения толщины пленок на подложках и анализа многослойных пленочных структур. Представлен обзор практических применений программного обеспечения TFCompanion (Semiconsoft) в составе измерительных комплексов MProbe ${ }^{\circledR}$ (Semiconsoft) для измерения и анализа отраженных спектров и расчета толщины толстых пленок с использованием встроенных алгоритмов быстрого преобразования Фурье (БПФ).

This paper presents examples of the software package intended to spectra treatment in order to determine thickness of films. Practical usage of TFCompanion (Semiconsoft) software as part of the MProbe ${ }^{\circledR}$ (Semiconsoft) measuring systems for reflected spectra's analysis and thin films' thickness calculation performed by embedded fast Fourier transform (FFT) algorithms is observed.

пектроскопическая рефлектометрия является одним из самых простых и недорогих инструментов неразрушающего контроля в тонкопленочной и толстопленочной технологии создания диэлектрических и тонких металлических слоев и устройств на их основе [1-4]. В настоящее время этот метод широко используется не только для анализа сформированных пленочных структур, но и для контроля в процессе формирования и обработки многослойных покрытий, как в процессах физического напыления слоев [2], так и в процессах реактивного и плазмохимического травления пленочных структур [3]. Этот метод позволяет точно и быстро выполнить измерение толщины и некоторых оптических констант нескольких последовательных слоев в сложных пленочных структурах. Развитие метода позволило с высокой точностью определять параметры не только диэлектрических слоев, но также и тонких металлических пленок [4].

В методе обычно используется измерение интенсивности отраженного излучения в широком диапазоне длин волн. В большинстве экспериментальных схем применяется неполяризованный свет, падающий под прямым углом. В зависимости от исследуемого материала могут 
использоваться различные диапазоны электромагнитного спектра: от глубокого ультрафиолета до дальнего ИК. Измерения состоят из двух последовательных этапов. На первом этапе, его часто называют калибровкой сигнала, используют образец подложки (например, чистый кремний), для которого известен коэффициент отражения, при этом проводят измерение отраженного сигнала от калибровочного образца. На следующем этапе измеряют интенсивность сигнала, отраженного от исследуемого образца, то есть подложки с пленкой. После этого вычисляется абсолютное значение коэффициента отражения в зависимости от длины волны. В качестве источника излучения обычно используют галогенную и/или дейтериевую лампы (для различных диапазонов длин волн). Излучаемый поток передается через волоконный световод и освещает исследуемый образец. Когда необходима малая освещаемая поверхность, применяют микрообъективы, которые фокусируют луч на участок площадью несколько микрон. Отраженный свет собирается в центральном волокне световода и направляется в спектрометр, оснащенный детектором на основе ПЗС или в длинноволновой области матричным детектором, обычно на основе InGaAs. Спектр снимается за несколько миллисекунд - в зависимости от требуемого соотношения сигнал/ шум.

Спектры отражения регистрируются и анализируются программными алгоритмами. В случае тонких пленок (менее 150-200 нм) измеренная кривая коэффициента отражения сравнивается с теоретической кривой, полученной для многослойной модели. Чтобы минимизировать

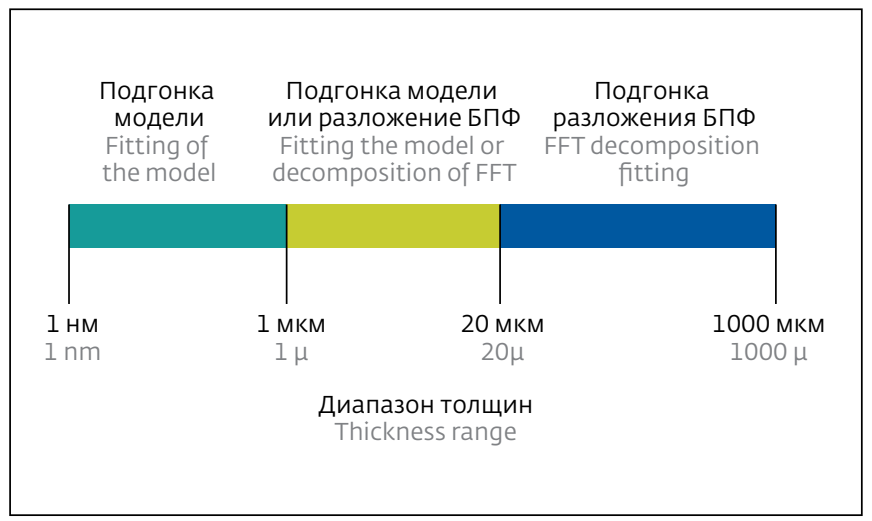

Рис.1. Диапазоны толщин пленок, определяющие границы применения способа подгонки параметров модели и БПФ Fig.1. Ranges of film thicknesses defining the boundaries of the application of the method of fitting model parameters and FFT

расхождение между измеренной и рассчитанной кривыми, программный алгоритм изменяет в параметрах модели толщину слоя. Модель состоит из описания параметров одного или нескольких слоев пленок. Для каждого слоя процедура начинается с оценки толщины, а затем задаются значения показателя преломления и коэффициента поглощения. Эти значения для большинства материалов содержатся во встроенной базе данных. Для новых или сложных по составу материалов данные величины можно оценить с помощью уравнений, описывающих зависимость $\mathrm{n}$ и $\mathrm{k}$ от длины волны. Когда измеряемые слои толстые, и отраженные спектры содержат множество пиков в диапазоне длин волн, используется быстрое преобразование Фурье (БПФ, алгоритм ускоренного вычисления дискретного преобразования

Таблица 1. Погрешности измерений при применении БПФ

Table T. Measurement errors when applying of FFT

\begin{tabular}{|c|c|c|c|c|c|}
\hline \multirow[b]{2}{*}{$\begin{array}{c}\text { Погрешность } \\
\text { измерений }\end{array}$} & \multirow[b]{2}{*}{ Длина волны } & \multicolumn{4}{|c|}{ Толщина пленки } \\
\hline & & 200 нм & 500 нм & 700 нм & 1000 нм \\
\hline Абсолютная & \multirow{2}{*}{ 400-1 000 Hм } & $92 \mathrm{HM}$ & $36 \mathrm{HM}$ & $6 \mathrm{HM}$ & $0,6 \mathrm{HM}$ \\
\hline Относительная, \% & & $46 \%$ & $7 \%$ & $0,9 \%$ & $0,06 \%$ \\
\hline Абсолютная & \multirow{2}{*}{ 400-1700 нм } & $49 \mathrm{HM}$ & $2 \mathrm{HM}$ & $1,2 \mathrm{HM}$ & $0,4 \mathrm{HM}$ \\
\hline Относительная, \% & & $24 \%$ & $0,4 \%$ & $0,017 \%$ & $0,04 \%$ \\
\hline
\end{tabular}






Рис. 2. Моделируемый спектр отражения (диапазон длин волн 400-1 000 нм) для пленок состава $\mathrm{SiO}_{2} / \mathrm{Si}$. Толщина оксида: 200, 500, 700 и 1000 нм (толщина оксида, coответствующая одному периоду в диапазоне видимого света, составляет примерно 320 нм)

Fig.2. Simulated reflection spectrum (wavelength range of 400-1000 nm) for $\mathrm{SiO}_{2}$ / Si films. Oxide thickness: 200, 500, 700 and $1000 \mathrm{~nm}$ (the oxide thickness corresponding to one period in the visible light range is approximately $320 \mathrm{~nm}$ )

Фурье) сигнала для определения частот, соответствующих оптической толщине каждого слоя. Затем, зная показатель преломления материала, можно определить физическую толщину слоя. Наибольшее распространение этот метод получил в микроэлектронике при анализе толстых пленок фоторезистов [5].

Оборудование MProbe ${ }^{\circledR}$ (измерительные системы) и ПО обработки данных TFCompanion компании Semiconsoft предназначены для работы с такими материалами, как оксиды,

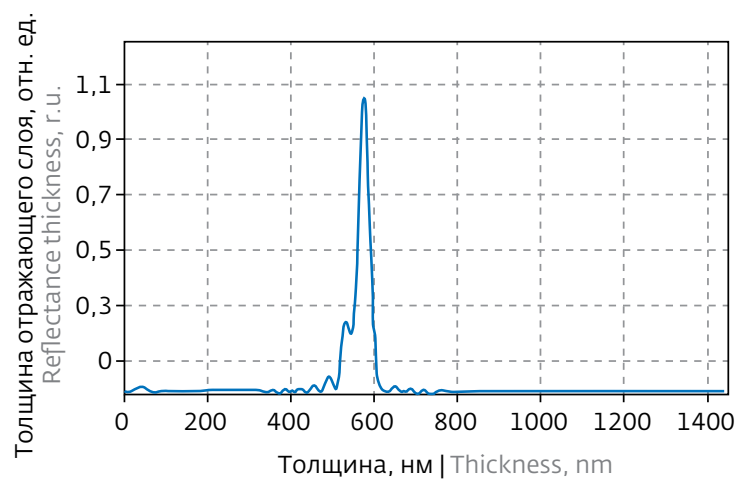

Рис.4. Использование дифференциального метода БПФ для измерения тонких пленок

Fig.4. Using the differential FFT method for measuring thin films

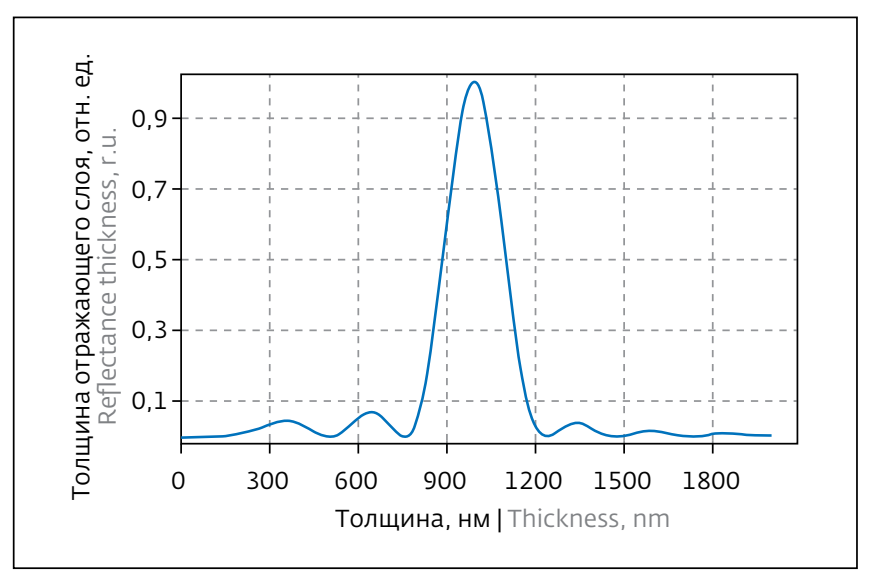

Рис.3. Толщина пленки оксида кремния, равная 1000 нм (диапазон длин волн 400-1 000 нм), определена с применением БПФ. Малая погрешность измерения (0,6 нм) показывает, что БПФ может быть успешно использовано для измерения пленок такой толщины

Fig.3. The silica film thickness of $1000 \mathrm{~nm}$ (the wavelength range of 400-1000 nm) was determined using FFT. The small measurement error $(0.6 \mathrm{~nm})$ indicates that the FFT can be successfully used to measure films of such thickness

нитриды, фоторезисты, полимеры, полупроводники (Si, a-Si, poly-Si), соединения полупроводников (AlGaAs, InGaAs, CdTe, CIGS), твердые покрытия ( $\mathrm{SiC}, \mathrm{DLC})$, полимерные покрытия (Парилен, РMMA, Полиамиды), тонкие пленки металлов (до 50 нм). Благодаря этому оборудованию можно быстро и достоверно измерить тонкопленочные гетероструктуры (TCO, CIGS, $\left.\mathrm{cdS}, \mathrm{CdTe}, \mathrm{ZnO} / \mathrm{Al}_{2} \mathrm{O}_{3}\right)$ с большим количеством слоев, применяющиеся при производстве солнечных элементов и органической электроники, сложных оптических систем, например, фильтров.

Система MProbe ${ }^{\circledR}$ способна измерять толщину практически любой прозрачной или полупрозрачной пленки в диапазоне от 1 нм до 1 мм.

Таблица 2. Сравнение результатов измерений тонкой пленки, полученных при использовании различных методов Table 2. Comparison of thin film measurements obtained using various methods

\begin{tabular}{|l|c|c|c|}
$\begin{array}{c}\text { Метод } \\
\text { измерения } \\
\text { толщины }\end{array}$ & $\begin{array}{c}\text { Подгонка } \\
\text { кривой }\end{array}$ & $\begin{array}{c}\text { Дифферен- } \\
\text { циальный } \\
\text { метод БПФ }\end{array}$ & $\begin{array}{c}\text { Метод прямого } \\
\text { использования } \\
\text { БПФ }\end{array}$ \\
\hline $\begin{array}{l}\text { Толщина } \\
\text { тонкого } \\
\text { слоя }\end{array}$ & $372,7 \mathrm{HM}$ & $375 \mathrm{HM}$ & $393,1 \mathrm{HM}$ \\
\hline
\end{tabular}




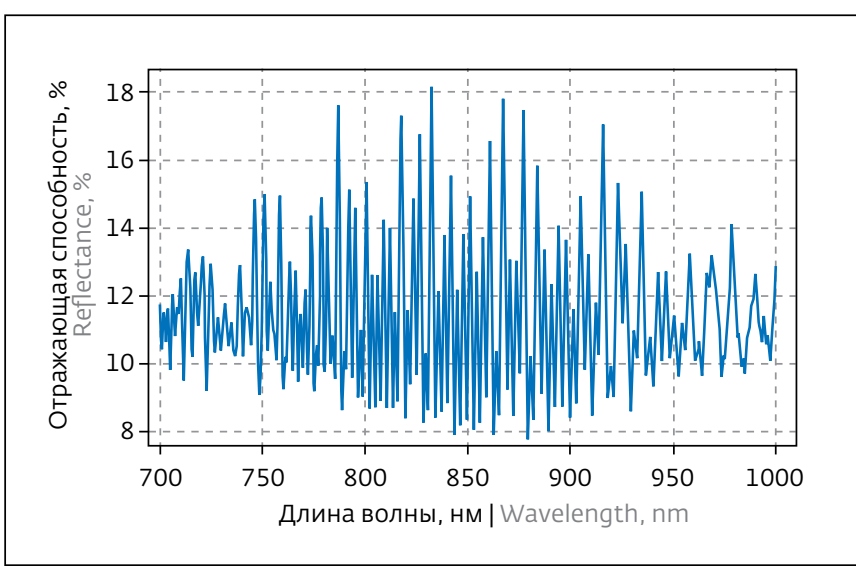

Pис.5. Спектр отражения, измеренный с помощью MProbeVisHR (диапазон длин волн 700-1 000 нм). Образец представляет собой четырехслойное полимерное покрыmuе (наклейку)

Fig.5. The reflection spectrum measured using MProbeVisHR (wavelength range of 700-1000 nm). The sample is a four-layer polymer coating (sticker)

\section{ПРАКТИЧЕСКОЕ ПРИМЕНЕНИЕ БПФ В СООТВЕТСТВИИ С ДИАПАЗОНОМ ТОЛЩИН ИЗМЕРЯЕМЫХ ТОНКИХ ПЛЕНОК}

Метод разложения БПФ - очень мощный и удобный инструмент, позволяющий быстро получить результаты, имеющий низкие требования к калибровке (например, дрейф/изменения интенсивности не влияют на результаты) и данным об измеренном образце. Основными недостатками разложения БПФ являются потребность в информации о показателе преломления и, до недавнего времени, - более низкая точность по сравнению с прямой подгонкой модели [6].

Недавние усовершенствования ПО ТFCompanion значительно улучшили точность и разрешающую способность алгоритма разложения БПФ. В большинстве случаев точность измерения толщин пленок составляет менее $0,1 \%$.

Применение БПФ основано на том, что интерференция в толстых пленках создает периодическую функцию интенсивности светового излучения в зависимости от длины волны. Диапазон длин волн, используемый для измерения, и показатель преломления материала определяют минимальную толщину пленок, для измерения которых применение БПФ имеет практический смысл. На рис.1 показана схема, позволяющая определить более подходящий метод анализа результатов измерений в зависимости от диапазона толщины пленок. Выбор одного периода или части периода функции приводит к ухудшению точности этого метода и накладывает



Pис.6. Анапиз данных разложения БПФ (см. рис.5). Положения пиков указывают толщину слоев

Fig.6. Analysis of FFT decomposition data (see Fig.5). Peak positions indicate layer thickness

ограничение на минимальную толщину пленок, которая может быть практически определена с помощью БПФ. В табл.1 показаны погрешности измерения с применением БПФ в зависимости от толщины пленок и диапазона длин волн [7].

Смоделированные спектры отражения оксидных пленок толщиной 200, 500, 700 и 1000 нм были проанализированы с использованием БПФ (рис.2, 3). В ходе анализа были вычислены ошибки измерения (абсолютные и относительные). Спектры были сгенерированы для длин волн 400-1000 нм и 400-1700 нм.

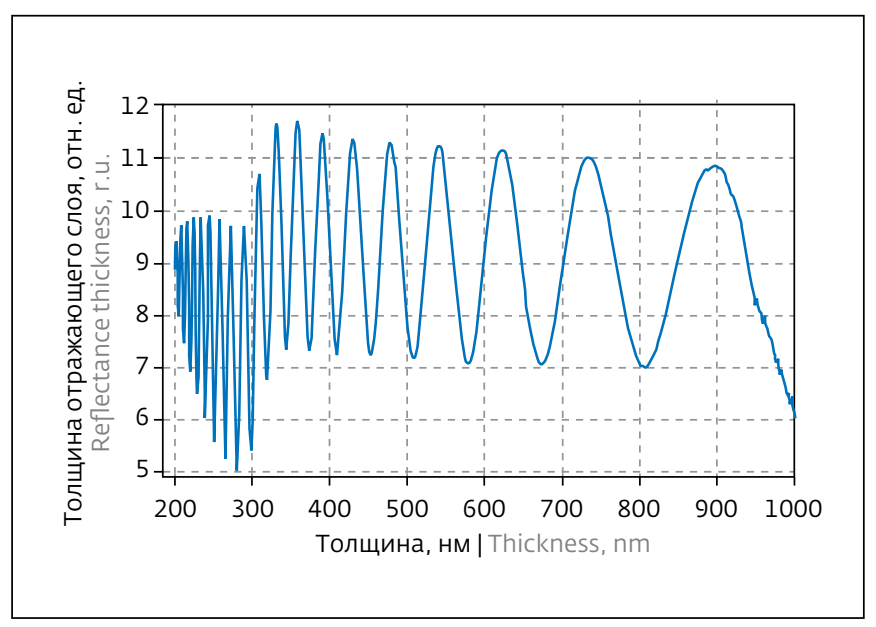

Рис.7. Спектр отражения слоя оксида алюминия на стекле, полученный с помощью системы MProbe UVVisSR (дuanaзон длин волн 200-1 000 нм)

Fig.7. The reflection spectrum of an aluminum oxide layer on glass, obtained using the MProbe UVVisSR system (wavelength range of 200-1000 nm) 


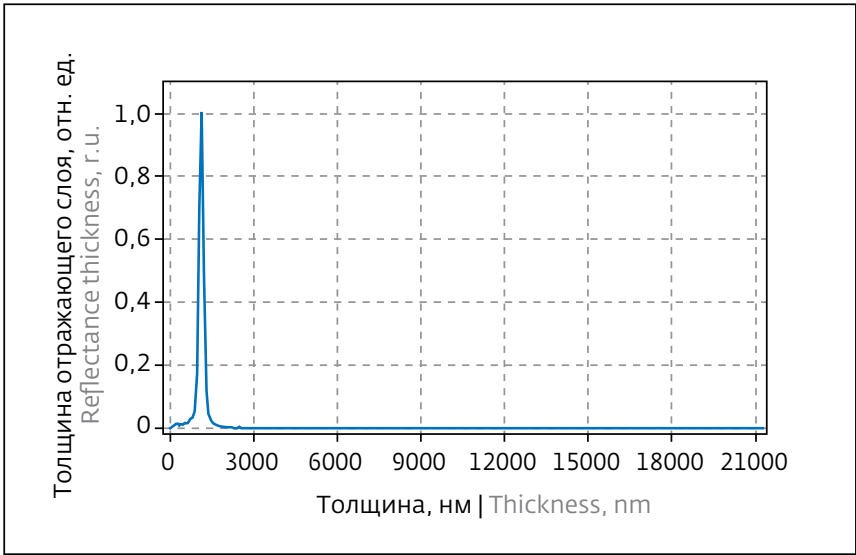

Рис.8. Разложение БПФ данных, представленных на рис.8, с использованием библиотеки свойств оксида алюминия. Положение пика указывает толщину слоя $1164 \mathrm{HM}$

Fig.8. Decomposition of the FFT data presented in Fig.8, using the library of properties of alumina. The peak position indicates the layer thickness of $1164 \mathrm{~nm}$

Прямое измерение тонких пленок с применением БПФ вызывает большие погрешности. Более того, во многих случаях измеряемый образец имеет толстый и тонкий слои. Например, стекло, покрытое 400 нм слоем оксида индия-олова и фоторезистом толщиной 5000 нм. Если в этом случае используется БПФ, можно видеть три пика: пик слоя 1 (L1), пик слоя 2 (L2) и пик общей толщины $(\mathrm{L} 1+\mathrm{L} 2)$. Это дает возможность определить толщину L1 (тонкой пленки) как разность между общей

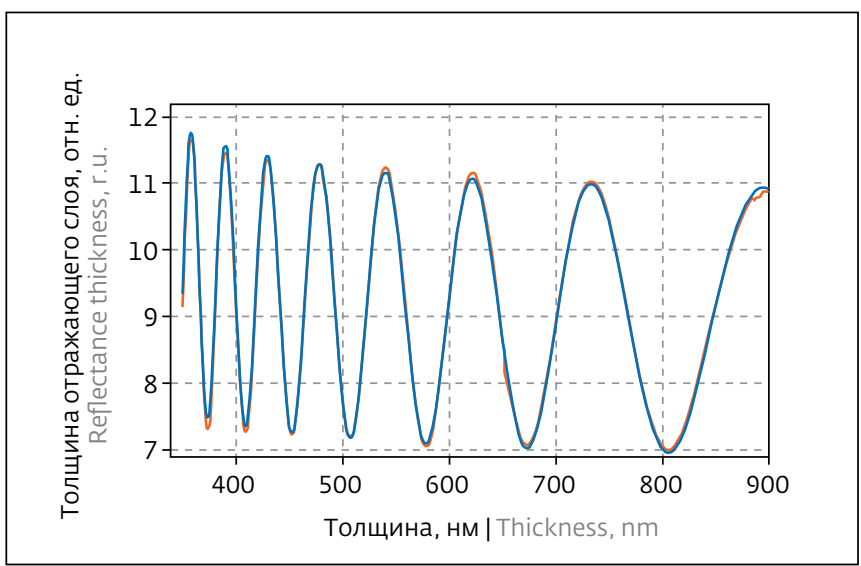

Рис.10. Прямая подгонка модели к измеренным данным для определения толщины и показателя преломления слоя оксида алюминия. Толщина слоя - 1202 нм

Fig.10. Direct fitting of the model to the measured data to determine the thickness and refractive index of the aluminum oxide layer. The layer thickness is $1202 \mathrm{~nm}$

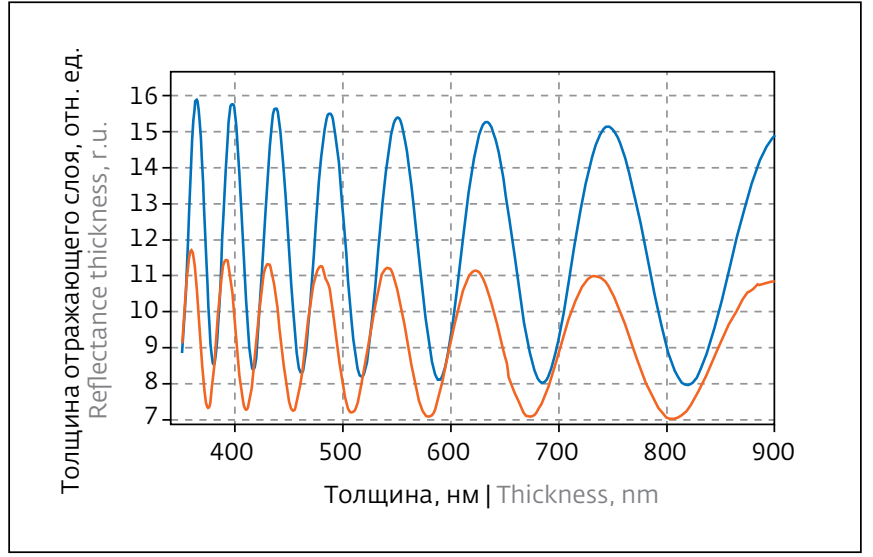

Рис.9. Прямое сравнение модели, построенной по результатам разложения БПФ (красный), и измеренных данных (синий) показывает, что показатель преломления опредепен неверно

Fig.9. A direct comparison of the model constructed from the results of the FFT decomposition (in red) and the measured data (in blue) shows that the refractive index is determined incorrectly

толщиной и толщиной слоя L2 (рис.4). Этот косвенный метод определения дифференциальной толщины дает гораздо более точный результат, чем прямое измерение.

\section{ПРАКТИЧЕСКИЕ ПРИМЕРЫ ИСПОЛЬЗОВАНИЯ МЕТОДА БПФ}

\section{Измерение многослойного полимерного покрытия}

Многослойные полимерные пленки применяются в самых различных областях, например, для

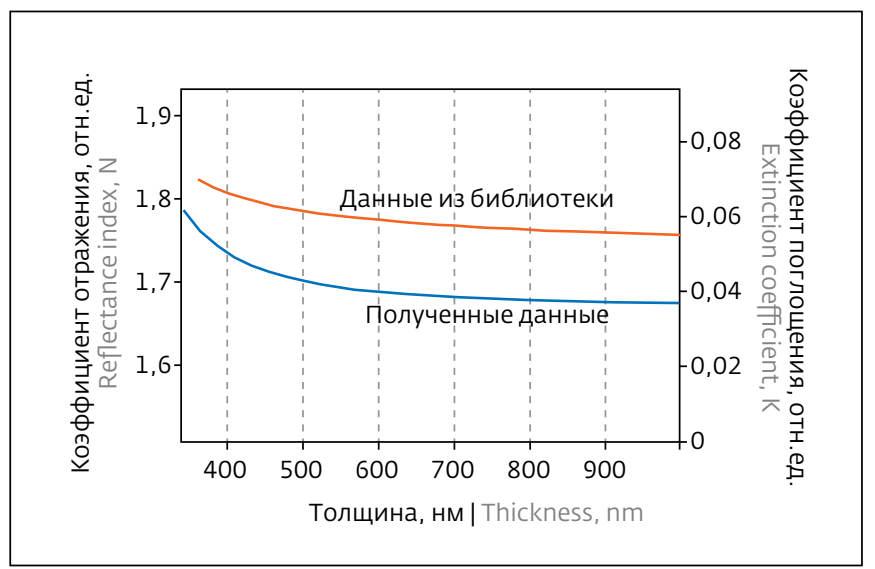

Рис.11. Сравнение показателя преломления, полученного по результатам измерений, и показателя преломления, взятого из библиотеки значений

Fig.11. Comparison of the refractive index obtained from the measurement results and the refractive index taken from the library of values 


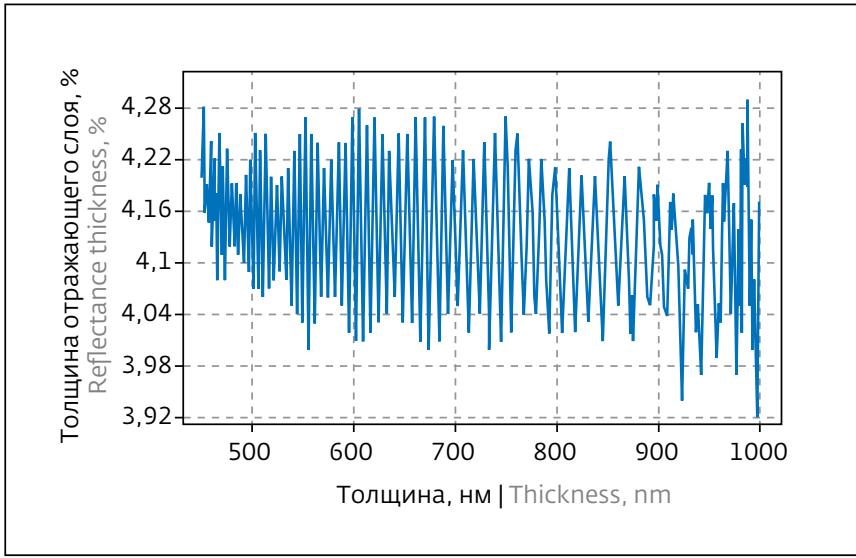

Рис.12. Спектры отражения поликарбоната с твердым покрытием (фара). Измерение выполнялось с помощью системы MProbe Vis (диапазон диин волн 4001000 HM)

Fig.12. The reflection spectra of polycarbonate with a hard coating (headlight). The measurement was performed using the MProbe Vis system (wavelength range of 400-1000 nm)

упаковки пищевых продуктов, изготовления наклеек/этикеток и т.д. [2].

В данном примере рассмотрим четырехслойное полимерное покрытие со следующей структурой: изолирующий слой/кремниевый слой/клейкий слой/пленка ПЭТ (подложка). Кремниевый слой представляет собой тонкую пленку (d менее 1 мкм), которая позволяет "отклеить" изолирующий слой, чтобы обнажить клейкий слой наклейки (ПЭТ-пленку).



Рис.14. Сравнение разрешения при использовании разно20 количества точек БПФ. Расстояние между соседними точками БПФ (отсчетами) определяет максимальную погрешность измерений

Fig.14. Comparison of resolution when using a different number of FFT points. The distance between adjacent FFT points (readings) determines the maximum measurement error

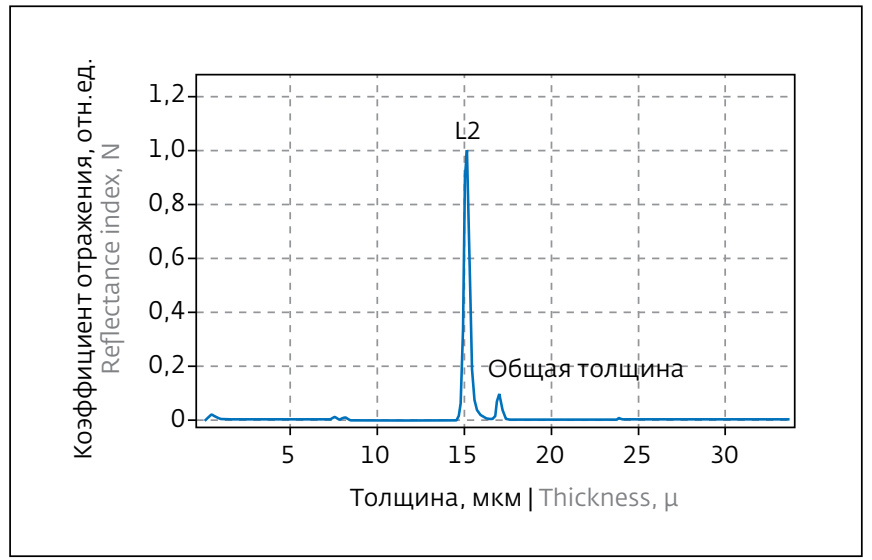

Рис.13. Разложение БПФ результатов измерений, приведенных на рис.15. Толщина IPL вычисляется с использованием функции определения дифференциальной толщины. Толщина твердого покрытия - 5,2 мкм, IPL - 1,85 мкм

Fig.13. FFT decomposition of the measurement results shown in Fig.15. The IPL thickness is calculated using the differential thickness function. The thickness of the solid coating $-5.2 \mu$, IPL $-1.85 \mu$

В результате анализа разложения БПФ получают пик для каждой границы раздела, положение этого пика указывает толщину слоя, а высота - "качество" границы раздела (рис. 5, 6). Следовательно, трехслойное покрытие должно иметь шесть пиков (последний пик - общая толщина). Пик кремниевого слоя ( $\left.3^{*}\right)$ не отображается отдельно (толщина слишком мала - менее 1 мкм), но отображается в комбинации с изолирующим

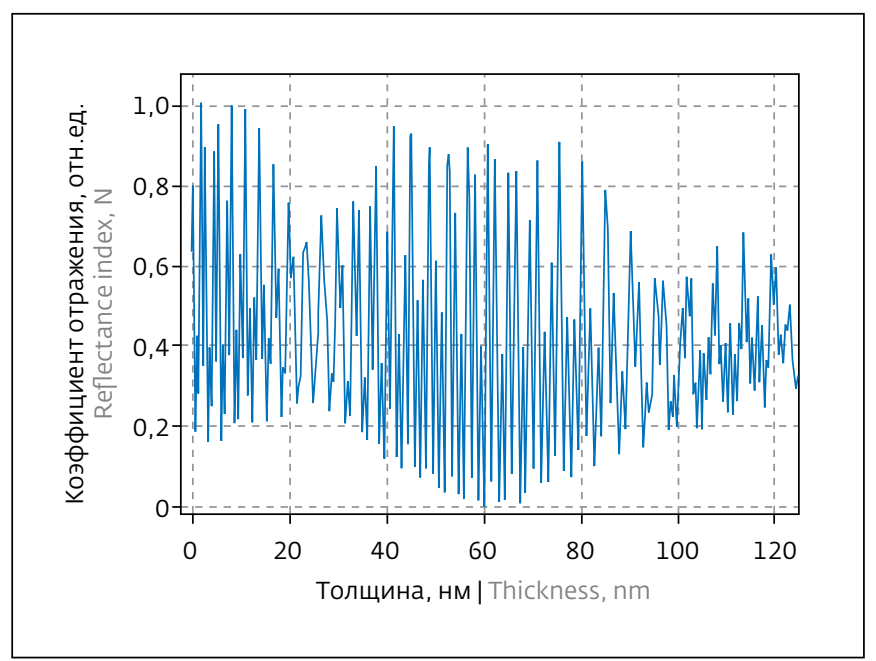

Рис.15. Измеренный спектр отражения покрытия (толщина примерно 18 мкм) на ПЭТ-пленке толщиной 38 мкм Fig.15. Measured reflectance spectrum of the coating (about $18 \mu$ thick) on a $38 \mu$ thick PET film 


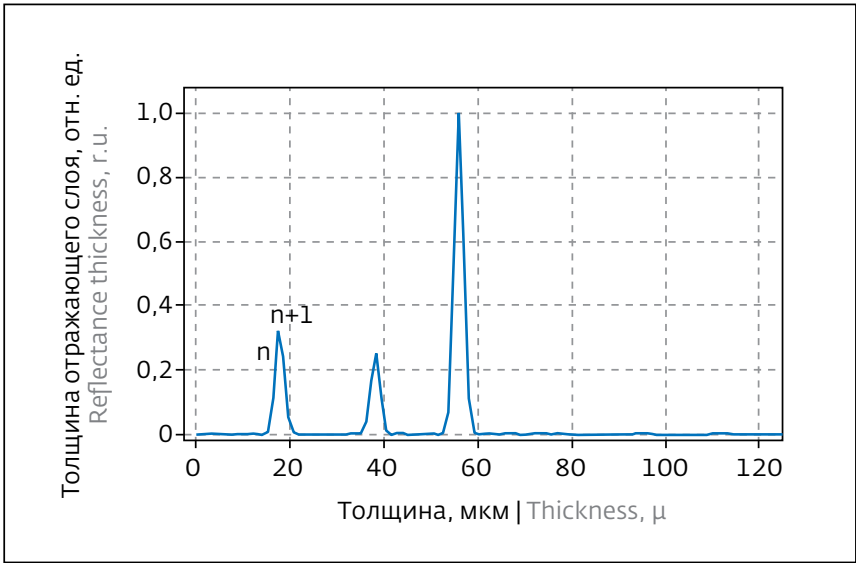

Рис.16. Пики, полученные с использованием разрешения по умолчанию (1 точка)

Fig.16. Peaks obtained using the default resolution (1 point)

и изолирующим + клейким слоями. Пик общей толщины также расширяется из-за слоя кремния. ПО MProbe TFCompanion способно автоматически идентифицировать пики и сопоставлять их со слоями. Эта функция помогает определить структуру измеряемой пленки и диапазон ожидаемых толщин. Однако, в большинстве случаев, эта информация также может быть получена из самого разложения БПФ и отправлена обратно в базу данных модели. Ограничения толщины слоя позволяют программе легко идентифицировать пики [2].

Разложение БПФ (см. рис.6) позволяет показать все слои (кроме слоя кремния). Часто некоторые из слоев оказываются скрытыми или соответствующий пик слишком мал для точного определения толщины слоя. В результате измерений получим:

- изолирующий слой - 36,8 мкм;

- кремниевый слой - 0,8 мкм;

- клейкий слой - 5,3 мкм;

- слой ПЭТ - 35,4 мкм.

\section{Измерение толщины слоя оксида алюминия (сапфира)}

Показатель преломления оксида алюминия, парилена, оксида иттрия и многих других соединений, образующих толстые пленки, зависит от условий осаждения. Использование библиотеки оптических свойств материалов совместно с БПФ может привести к неточному результату (рис.7, 8).

Сравнение модели и полученных данных с учетом результатов разложения БПФ показывает, что значение показателя преломления, используемое в модели - неточное (рис.9).



Рис.17. Пики, полученные с использованием высокого разрешения (4 точки)

Fig.17. High resolution peaks (4 points)

\section{Твердые покрытия}

Твердые покрытия применяются при осаждении на поверхность поликарбонатных изделий (линзы для очков, передние/задние фары). При нанесении твердое покрытие создает внутренний проникающий слой (IPL - inter penetration layer) в поликарбонате. IPL, как правило, довольно тонкий (d 1 мкм). Для контроля качества необходимо измерять толщину слоев IPL и твердых покрытий (рис.12, 13).

Значение показателя преломления IPL занимает промежуточное значение между показателями преломления поликарбоната и твердого покрытия, и оптический контраст между IPL и обоими материалами является слабым. В результате прямой пик IPL очень мал (на уровне шума). Функция дифференцирования толщины для определения IPL дает точные результаты.

\section{ПОВЫШЕНИЕ ТОЧНОСТИ АНАЛИЗА БПФ УВЕЛИЧЕНИЕМ РАЗРЕШЕНИЯ}

Точность измерения толщины пленок с помощью алгоритма БПФ зависит от точности определения положения пика. Максимальная

Таблица 3. Максимапьно возможная абсолютная погрешность измерения в зависимости от разрешения

Table 3. Maximum possible absolute measurement error depending on resolution

\begin{tabular}{|l|c|c|c|c|}
\hline \multicolumn{1}{|c|}{ Разрешение } & $\mathbf{1}$ & $\mathbf{2}$ & $\mathbf{3}$ & $\mathbf{4}$ \\
\hline $\begin{array}{l}\text { Максимальная } \\
\text { возможная } \\
\text { погрешность, мкм }\end{array}$ & 0,548 & 0,274 & 0,137 & 0,068 \\
\hline
\end{tabular}




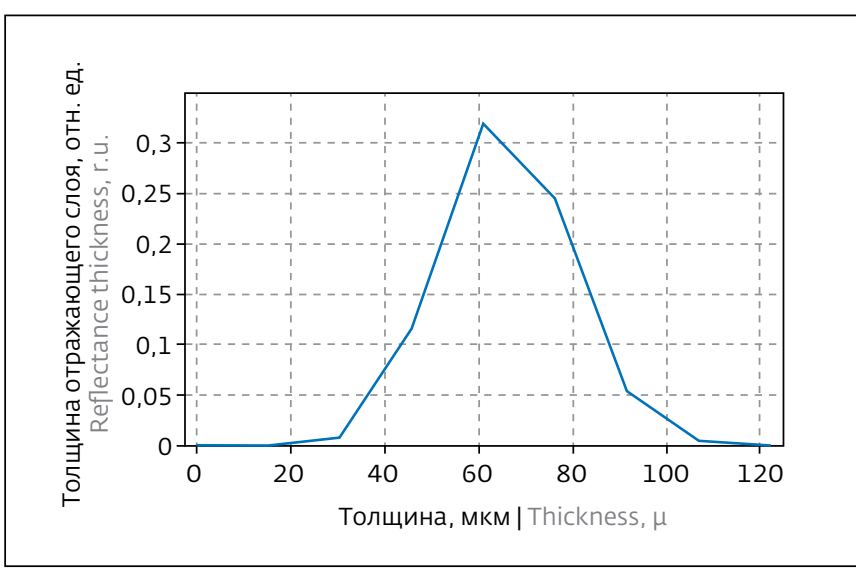

Рис.18. Фрагмент пика, приведенного на рис.16 (разрешение по умолчанию - 1 точка)

Fig.18. Fragment of the peak shown in Fig.16 (default resolution is 1 point)

погрешность измерения может быть определена как $\left(\mathrm{X}_{\mathrm{n}+1}-\mathrm{X}_{\mathrm{n}}\right) / 2$, где $\mathrm{X}_{\mathrm{n}+1}$ и $\mathrm{X}_{\mathrm{n}}$ являются точками (отсчетами), соседними с пиком (рис.14).

Повысить точность можно увеличением количества точек, то есть разрешения. Этот вариант доступен в ПО ТFCompanion и рассматривается ниже на примере покрытия полимерной пленки (рис.15). Рассмотрим покрытие (d 18 мкм) на ПЭТпленке толщиной 38 мкм.

Точки БПФ распределяются в максимальном диапазоне измеряемой толщины, но часто интерес представляет только часть диапазона толщины. В нашем примере максимальная измеряемая толщина составляет 280 мкм, а толщина образцов- 18 и 38 мкм. Можно увеличить количество точек, используя метод передискретизации.

В данном ПО можно регулировать разрешение от 1 до 5. Результат влияния представлен на рис.16-19 и табл.3.

\section{ЗАКЛЮЧЕНИЕ}

Спектроскопическая рефлектометрия является простым и неразрушающим методом, который может использоваться для широкого спектра применений: измерение толщин и оптических постоянных. ПО Semiconsoft - хороший инструмент, позволяющий быстро получить необходимую информацию о покрытиях и тонких пленках. Установки MProbe могут эффективно использоваться для тонкопленочных изделий, например, в НИОКР, производстве, мониторинге, а также для многих других задач для обеспечения высокой точности толстопленочных технологий.



Рис.19. Фрагмент пика, приведенного на рис.17 (высокое разрешение - 4 точки)

Fig.19. Fragment of the peak shown in Fig.17 (high resolution -4 points)

\section{ЛИТЕРATУPA / REFERENCE}

1. Optical thin films and coatings. From materials to applications. Edited by Angela Piegari and François Flory, 2013 // Ch.3. Optical monitoring strategies for optical coating manufacturing, TIKHONRAVOV A.V., TRUBETSKOV M.K. , AMOTCHKINA T.V.

2. Tikhonravov A.V., Trubetskov M.K., Amotchkina T.V. DeBell G., Pervak V., Krasilnikova A., Sytchkova M., Grilli L., Ristau D. Optical parameters of oxide films typically used in optical coating production // APPLIED OPTICS. 2011. Vol. 50. No. 9 / 20. P. 75-85.

3. Stutzman B.S., Huang H.T., Terry F.L. Two-channel spectroscopic reflectometry for in situ monitoring of blanket and patterned structures during reactive ion etching // J. Vac. Sci. Technol. 2000. Vol. 186. P. 2785.

4. Amotchkina T.V., Janicki V., Sancho-Parramon J., Tikhonravov A.V., Trubetskov M.K., Zorc H. General approach to reliable characterization of thin metal films // APPLIED OPTICS. 2011. Vol. 50. No. 10 / 1. P. 1453.

5. Корольков В.П., Конченко А.С., Черкашин В.В., Миронников Н.Г. Разработка методов формирования и контроля заданного распределения толщины фоторезиста при изготовлении конформальных корректоров // Компьютерная оптика. 2016. T. 40. № 4. C. 482-488.

6. Asinovski L. Optical metrology: performance analysis: approach and metrics, Characterization and Metrology for ULSI Technology, AIP conference proceedings 788, p. 314 (2005).

7. Asinovski L. Performance analysis of ellipsometer systems, Thin Solid Films, 455-6, p. 790 (2004). 